\title{
ASSESSMENT OF COAGULATION PARAMETERS IN CIRRHOSIS OF LIVER
}

\author{
Chikkalingaiah ${ }^{1}$, Keshava H. K², Prakruthi J3
}

\section{HOW TO CITE THIS ARTICLE:}

Chikkalingaiah, Keshava H. K, Prakruthi J. "Assessment of Coagulation Parameters in Cirrhosis of Liver”. Journal of Evolution of Medical and Dental Sciences 2014; Vol. 3, Issue 10, March 10; Page: 2595-2602, DOI: $10.14260 /$ jemds/2014/2179

ABSTRACT BACKGROUND: Cirrhosis is a pathologically defined entity that is associated with a spectrum of characteristic clinical manifestations associated with major hemostatic abnormalities. Cirrhosis encompasses both pro-coagulant as well as anti-coagulant tendencies. These fragile coagulation cascade may result in life threatening bleeding manifestations. AIMS: To determine the relationship between coagulation abnormalities and the stage of Cirrhosis of liver. To assess the ability of prolonged prothrombin time in predicting the presence of encephalopathy. METHODS AND MATERIALS: This was a 2 year observational study performed in KIMS Hospital, Bangalore involving 50 patients of cirrhosis of liver without prior history of any bleeding manifestations. The patients underwent detail history and clinical examination with routine biochemical and coagulation parameter evaluation with Ultrasound of abdomen. Child Pugh's grade was calculate for all patients and the results were tabulated. STASTISTICAL ANALYSIS: SPSS version 17 was used for data processing purpose. One way ANOVA test was used for assessing relationship of PT and APTT with Child Pugh's Grade. Pearsons chi square test was applied for relationship of encephalopathy and coagulation parameters. RESULTS: In our study the prothrombin time was prolonged in 27 patients (54\%) and APTT was prolonged in 39 patients (78\%). The mean prothrombin time in our study was 24.328 \pm 8.54 , and mean APTT was 46.366 \pm 7.54 , which was significant. The mean PT prolongation in Grade C Child Pugh's grade among 34 patients is $12.82 \pm 9.07$ as compared to Grade A which had a mean of $1.15 \pm 0.78$. On performing one way ANOVA for PT: $F(2,49)=5.68, p>0.01$. The mean prolongation of APTT in Grade C cirrhosis among 34 patients is $18.42 \pm 7.32$ as compared to Grade A cirrhosis which had a mean of $18.20 \pm 16.69$. On performing one way ANOVA for APT: $F(2,49)=4.41$. On performing Pearson Chi square test was performed, for PT and encephalopathy was 0.497, similarly for APTT and encephalopathy was 0.472 which were statistically significant. CONCLUSION: Prolongation of coagulation parameters is associated with higher grades of cirrhosis and also associated with poor prognosis. There is no role of coagulation parameters in predicting the presence of encephalopathy in cirrhosis of liver.

KEYWORDS: Cirrhosis of liver, Prothrombin time, Activated partial thromboplastin time, Coagulation abnormalities

INTRODUCTION: Cirrhosis is a pathologically defined entity that is associated with a spectrum of characteristic clinical manifestations. ${ }^{1}$ The cardinal pathologic features reflect irreversible chronic injury of the hepatic parenchyma and extensive fibrosis in association with the formation of regenerative nodules. There are major alterations in the hemostatic pathways in most patients with liver disease including altered platelet and endothelial function, altered clotting factors and conditions such as hyperfibrinolysis, dysfibrinogenemia and renal failure which may be superimposed on these underlying abnormalities. ${ }^{2}$ Cirrhosis encompasses both pro-coagulant as well as anti-coagulant tendencies. ${ }^{3}$ 
The main clinical importance of these coagulation disorders lies in the fact that the ability of the fragile coagulation system to tolerate or recover from an insult is markedly impaired in cirrhotic, easily tipped into a state favoring either hemorrhage or less frequently thrombosis. ${ }^{4}$ Also the prevalence of cirrhosis of liver in our country has been on the rise owing to the increased alcohol abuse in the population, currently the extrapolated prevalence is 1,566,280.5 This is associated with increased incidence of bleeding tendencies due to portal hypertension and as well as coagulation abnormalities. The objective of this study was to evaluate coagulation abnormalities in patients with liver cirrhosis.

OBJECTIVE: To determine the relationship between coagulation abnormalities and the stage of Cirrhosis of liver. To assess the ability of prolonged prothrombin time in predicting the presence of encephalopathy.

\section{MATERIALS AND METHODS:}

- Type of Study: Observational study.

- Sample: Dept. of Medicine, Outpatient department and Inpatients, KIMS Hospital, Bangalore.

- Duration of study: Oct 2011 - Sept 2013.

- Sample size:50

Inclusion Criteria: Patients with cirrhosis of liver without any past history of upper (or) lower gastrointestinal bleed were included in the study.

Diagnosis of cirrhosis was based on a combination of history, clinical findings, impaired liver function tests and abdominal ultrasound.

\section{Exclusion Criteria:}

- Patients with present or previous history of GI bleed.

- Patients who have undergone sclerosis (or) band ligation of esophageal varices, TIPSS (or) surgery for portal hypertension.

- Patients who are on drugs which are definitely associated with thrombocytopenia will be excluded.

Fifty patients with Cirrhosis of liver, attending the medical wards and outpatient departments of Kempegowda Institute of Medical Sciences and Research Institute, Bangalore between the months of November 2011 to October 2013 were selected, based on inclusion and exclusion criteria. Informed consent was taken from all patients (or next of kin in case patient is unconscious).

All patients in the study underwent a full clinical evaluation .Clinical history and physical examination findings were recorded with particular attention to present or previous hematemesis, malena, bleeding per rectum, bleeding tendencies, alcoholism, blood transfusion, intake of hepatotoxic drugs, exposure to Sexually transmitted diseases, IV drug abuse, jaundice, anaemia, edema, stigmata of chronic liver disease, dilated abdominal veins, ascites, splenomegaly and encephalopathy (with West Haven grading).

All patients underwent biochemical tests, like liver function tests, complete blood counts, renal function tests, PT with INR, APTT, and ultrasonography of the abdomen to confirm the presence 
of cirrhosis. Child-Pugh score was calculated for all patients. Upper GI endoscopy was done in all patients to look for the presence of varices and signs of bleeding.

All the data was collected on predesign proforma and statistical package for social sciences (SPSS) version 17 was used for data processing purpose. Frequencies and means \pm Standard.

Deviation of data like age PT and APTT was calculated. All values considered significant when $\mathrm{p}$ value is 0.05. One way ANOVA test was used for assessing relationship of PT and APTT with Child Pugh's Grade. Pearson's chi square test was applied for relationship of encephalopathy and coagulation parameters.

RESULTS: The study was performed over a period of two years in the Dept. of Medicine, KIMS Hospital, Bangalore. It involved 50 patients of whom $86 \%$ were males (43 patients) and 14\% were females (7 patients). The distribution of age showed incidence of cirrhosis was maximum in age group of 51-60 years followed by 31-40 years. Distribution of age and gender has been shown in Figure 1 . Among 50 patients studied, features of encephalopathy were present in $22 \%$ of patients $(11$ patients). The clinical signs noted among the patients were most commonly Ascites constituting 88\% and Pedal edema along with Icterus accounting to about $86 \%$ each. The mean prothrombin time in our study was $24.328 \pm 8.54$, which was significant. In our study the prothrombin time was prolonged in 27 patients (54\%) and APTT was prolonged in 39 patients $(78 \%)$. The mean value of APTT in our study was $46.366 \pm 7.54$, which was significant.

On comparison of the prolongation of the PT and APTT in seconds with the grades of cirrhosis (Table 3) shows that the mean of the prolongation of PT in seconds shows an increasing trend with increasing grades of cirrhosis. The mean PT prolongation in Grade C Child Pugh's grade among 34 patients is $12.82 \pm 9.07$ as compared to Grade A which had a mean of $1.15 \pm 0.78$. This difference is statistical significant. On performing one way ANOVA for PT: F $(2,49)=5.68, p>0.01$ which was statistically significant suggesting that the greater the prolongation of the PT the higher the grade of Cirrhosis. Similarly there was an association with the higher grades of Cirrhosis with greater prolongation of APTT. The mean prolongation of APTT in Grade C cirrhosis among 34 patients is $18.42 \pm 7.32$ as compared to Grade A cirrhosis which had a mean of $18.20 \pm 16.69$. Although the mean value in the groups is same there is a significant variation in the standard deviation and hence the above values were statistically significant. On performing one way ANOVA for APT: F $(2,49)=4.41$, p $>0.01$ which was statistically significant suggesting that the greater the prolongation of the APTT the higher the grade of Cirrhosis.

On performing Pearson Chi square test was performed, for PT and encephalopathy was 0.497 which was not statistically significant (Table 5), similarly for APTT and encephalopathy was 0.472 which was also not statistically significant (Table 6).

DISCUSSION: Bleeding is a common clinical problem in patients with liver disease. Although it is generally believed to result from an inherent hemostatic defect, this concept has recently been challenged as changes occur in both pro- and antihemostatic pathways such that the net result is often balanced in many respects. ${ }^{2}$ Hemostasis is intimately related to liver functions, because most coagulation factors are synthesized in liver parenchymal cells and the liver's reticuloendothelial system serves an important role in the clearance of activation products. The extent of coagulation abnormalities depends upon the degree of disturbed liver function. ${ }^{6}$ 
Patients with cirrhosis suffer from a complex hemostatic disturbance, due to abnormalities in clotting and fibrinolytic system activation and in primary haemostasis. ${ }^{7}$

Coagulation and hemostasis in the liver failure patient is influenced by multiple, often opposing, and sometimes changing variables and may even produce a hypercoagulablestate. ${ }^{2}$

Coagulation indices because of their relationship to liver synthetic function are well established as prognostic markers in a variety of settings in both acute and chronic liver disease. ${ }^{8}$ On the basis of an extensive body of literature, these coagulation markers are clearly helpful in developing the prognosis, but the issue of test reproducibility is a concern. ${ }^{2}$

Our study included 50 patients diagnosed with cirrhosis of liver, who were analyzed for coagulation parameters. The distribution of age showed incidence of cirrhosis was maximum in age group of 51-60 years followed by 31-40 years. Among the patients studied males predominate the study population with $86 \%$ with females accounting for only $14 \%$. The mean prothrombin time in our study was $24.328 \pm 8.54$ which was significant.

In our study the prothrombin time was prolonged in 27 patients (54\%). Our results are in agreement with the results of other studies like, Satea et al, according to which cirrhotic patients had elevated prothrombin time ${ }^{9}$; Elgari MM also showed an increase in PT along with APTT10; Ahmed et al shows prolonged prothrombin time in cirrhotic patients (mean+SD=27.82 $+15.9 \mathrm{sec}$ ) ${ }^{11}$; Bernardl M., 1984 and Chisholm, M., 1992.12,13

In our study we identified prolonged APTT in 39 patients (78\%). The mean value of APTT in our study was $46.366 \pm 7.54$ which was significant. Our results are in agreement with the results of Saatea et al, which $77.5 \%$ cirrhotic patients had elevated APTT $^{9}$. Other studies also reported a prolonged APTT in cirrhotic patients, one study conducted by Ahmed et al shows prolonged APTT in cirrhotic patients (mean+SD $=52.76+21.95 \mathrm{sec}) \cdot{ }^{11}$

On comparison of the prolongation of the PT and APTT in seconds with the grades of cirrhosis (Table 3) shows that the mean of the prolongation of PT in seconds shows an increasing trend with increasing grades of cirrhosis. The mean PT prolongation in Grade C Child Pugh's grade among 34 patients is $12.82 \pm 9.07$ as compared to Grade A which had a mean of $1.15 \pm 0.78$. This difference is statistical significant. Similarly there was an association with the higher grades of Cirrhosis with greater prolongation of APTT. The mean prolongation of APTT in Grade C cirrhosis among 34 patients is $18.42 \pm 7.32$ as compared to Grade A cirrhosis which had a mean of $18.20 \pm 16.69$. Although the mean value in the groups is same there is a significant variation in the standard deviation and hence the above values were statistically significant. These findings were in agreement with various previous studies. ${ }^{9-12}$ Although these studied showed that there was prolongation of PT associated with cirrhosis of liver; performing ANOVA we were able to deduce that the prolongation of PT and APTT with the grades of cirrhosis of liver was directly proportional. Thus higher grades of cirrhosis of liver were associated with greater prolongation of PT and APTT.

Cirrhotic patients showed significant prolongation of PT and APTT time in the current study. This finding was compatible with the previous studies.7,12 Prothrombin time is commonly increased in liver diseases because liver is unable to manufacture adequate amount of clotting factors including those involved in extrinsic pathway. ${ }^{12}$ Out of factors II, V, VII and X, factor VII is the rate limiting factor in this pathway and thus has the greatest influence on PT. Fall of factor VII which has shortest half-life (6 hours) has poor prognosis. As the liver function worsens, the APTT may become abnormal, the reason being that factors IX, XI and XII and fibrin stabilizing factors are also produced by the 
liver. ${ }^{14}$ Prolongation of both PT and APTT may be noted while other biochemical tests of liver function in liver disease are still normal. Occasionally APTT may be abnormal when the PT is within the normal range. ${ }^{15}$

The ability of the coagulation parameters in predicting the presence of encephalopathy was also assessed. On performing Pearson Chi square test was performed, for PT and encephalopathy was 0.497 which was not statistically significant (Table 5), similarly for APTT and encephalopathy was 0.472 which was also not statistically significant (Table 6). Hence there is no role for the coagulation parameters in predicting the presence of encephalopathy.

CONCLUSION: PT was prolonged in 54\% of patients and APTT was prolonged in $78 \%$ which was significant statistically in cirrhosis of liver. The more the prolongation of prothrombin time the higher the grade of cirrhosis of liver is noted which was statistically significant. Similarly there was an association with the higher grades of Cirrhosis with greater prolongation of APTT. Thus prolongation of coagulation parameters is associated with higher grades of cirrhosis and also associated with poor prognosis. There is no role of coagulation parameters in predicting the presence of encephalopathy in cirrhosis of liver.

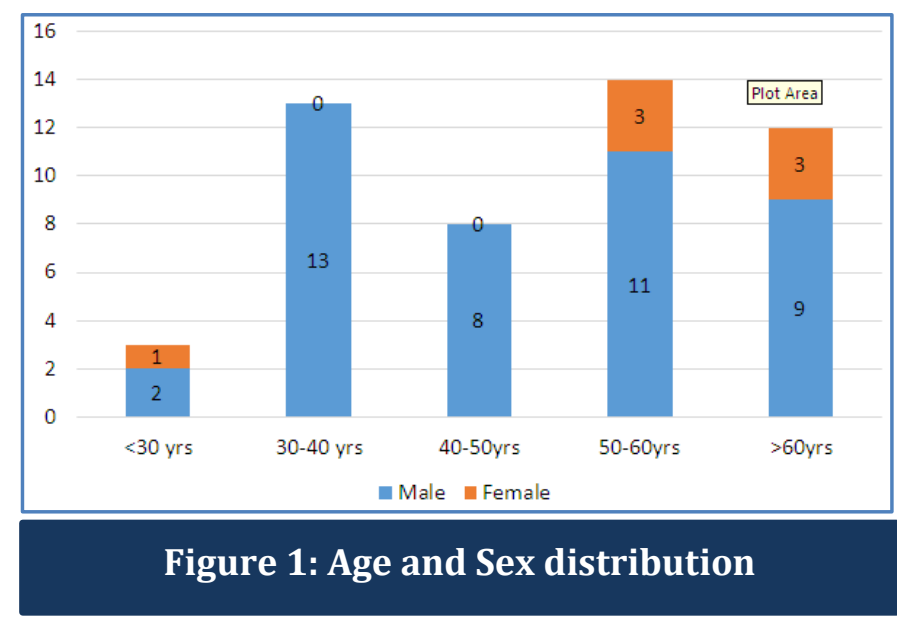

\begin{tabular}{|c|c|c|}
\hline Prothrombin time & Number of patients & Percentage \\
\hline Normal & 23 & $46 \%$ \\
\hline Prolonged & 27 & $54 \%$ \\
\hline 5-10 seconds & 5 & $10 \%$ \\
\hline $10-15$ seconds & 15 & $30 \%$ \\
\hline $15-20$ seconds & 2 & $4 \%$ \\
\hline$>20$ seconds & 5 & $10 \%$ \\
\hline Total & 50 & $100 \%$ \\
\hline
\end{tabular}




\begin{tabular}{|l|c|c|}
\hline \multicolumn{1}{|c|}{$\begin{array}{c}\text { Activated partial } \\
\text { thromboplastin time }\end{array}$} & $\begin{array}{c}\text { Number of } \\
\text { patients }\end{array}$ & Percentage \\
\hline Normal & 11 & $22 \%$ \\
\hline Prolonged & 39 & $78 \%$ \\
\hline 10-20seconds & 25 & $50 \%$ \\
20-30 seconds & 12 & $24 \%$ \\
$>20$ seconds $\quad$ Total & 2 & $4 \%$ \\
\hline \multicolumn{1}{|c|}{} & $\mathbf{5 0}$ & $\mathbf{1 0 0 \%}$ \\
\hline
\end{tabular}

Table 2: Cirrhotic patients in relation to activated partial thromboplastin time

\begin{tabular}{|c|c|c|c|c|}
\hline Coagulation parameter & Grades of Cirrhosis & N & Mean & S.D. \\
\hline \multirow{4}{*}{ Prothrombin Time } & $\mathrm{A}$ & 2 & 1.1500 & 0.77782 \\
\cline { 2 - 5 } & $\mathrm{B}$ & 14 & 5.5786 & 3.50037 \\
\cline { 2 - 5 } & $\mathrm{C}$ & 34 & 12.8235 & 9.07314 \\
\cline { 2 - 5 } & Total & $\mathbf{5 0}$ & $\mathbf{1 0 . 3 2 8 0}$ & $\mathbf{8 . 5 3 8 6 5}$ \\
\hline \multirow{3}{*}{ Activated partial thromboplastin time } & $\mathrm{A}$ & 2 & 18.2000 & 16.68772 \\
\cline { 2 - 5 } & $\mathrm{B}$ & 14 & 11.8357 & 4.58402 \\
\cline { 2 - 5 } & $\mathrm{C}$ & 34 & 18.4176 & 7.31812 \\
\cline { 2 - 5 } & Total & $\mathbf{5 0}$ & $\mathbf{1 6 . 5 6 6 0}$ & $\mathbf{7 . 4 9 7 1 2}$ \\
\hline
\end{tabular}

Table 3: Correlation between the grades of cirrhosis and Coagulation parameters (prolongation in seconds)

\begin{tabular}{|l|c|c|c|c|}
\hline \multicolumn{2}{|c|}{} & df & F & Sig. \\
\hline \multirow{3}{*}{ PT } & Between Groups & 2 & 5.686 & .006 \\
\cline { 2 - 5 } & Within Groups & 47 & & \\
\cline { 2 - 5 } & Total & $\mathbf{4 9}$ & & \\
\hline \multirow{3}{*}{ APT } & Between Groups & 2 & 4.410 & .018 \\
\cline { 2 - 5 } & Within Groups & 47 & & \\
\cline { 2 - 5 } & Total & $\mathbf{4 9}$ & & \\
\hline
\end{tabular}

Table 4: One way ANOVA in Child Pugh's Grades with PT and APTT

\begin{tabular}{|c|c|c|c|c|c|}
\hline \multicolumn{6}{|c|}{ Chi-Square Tests } \\
\hline & Value & Df & $\begin{array}{c}\text { Asymp. Sig. } \\
\text { (2-sided) }\end{array}$ & $\begin{array}{l}\text { Exact Sig. } \\
\text { (2-sided) }\end{array}$ & $\begin{array}{l}\text { Exact Sig. } \\
\text { (1-sided) }\end{array}$ \\
\hline Pearson Chi-Square & 0.119 & 1 & 0.730 & & \\
\hline & & & & 1.000 & 0.497 \\
\hline $\mathrm{N}$ of Valid Cases & 50 & & & & \\
\hline
\end{tabular}




\begin{tabular}{|c|c|c|c|c|c|}
\hline \multicolumn{7}{|c|}{ Chi-Square Tests } \\
\hline & Value & Df & $\begin{array}{c}\text { Asymp. Sig. } \\
\text { (2-sided) }\end{array}$ & $\begin{array}{c}\text { Exact Sig. } \\
\text { (2-sided) }\end{array}$ & $\begin{array}{c}\text { Exact Sig. } \\
\text { (1-sided) }\end{array}$ \\
\hline Pearson Chi-Square & 0.262 & 1 & 0.609 & & \\
\hline & & & & 1.000 & 0.472 \\
\hline N of Valid Cases & 50 & & & & \\
\hline
\end{tabular}

Table 6: Activated partial thromboplastin time and presence of encephalopathy

\section{REFERENCES:}

1. Bruce R. Bacon. Cirrhosis and its complications. Chapter 308. Harrison's principles of Internal Medicine 18th edition. McGraw-Hill. 2012

2. Coagulation Disorders and Hemostasis in Liver Disease: Pathophysiology and Critical Assessment of Current Management. Hepatology, vol. 44, no. 4, 2006

3. Tripodi, A. Hemostasis abnormalities in liver cirrhosis: myth or reality? Pol Arch Med Wewn 2008, 118(7-8):445-8.

4. Hayat, A.S., N. Shaikh and F. Memon. Identification of Precipitating Factors in Hepatic Encephalopathy Patients at Liaquat University and Hospital Jamshoro. World Applied Sciences J 2010, 8(6): 661-666.

5. US Census Bureau, International Data Base, 2004.

6. Mammen E.F. Coagulation abnormalities in liver disease. Hematol Oncol Clin North Am 1992, 6(6):1247-57.

7. Violi, F., R. Leo, E. Vezza, S. Basili, C. Cordova and F. Balsano. Bleeding time in patients with cirrhosis: relation with degree of liver failure and clotting abnormalities. J Hepatol 1994, 20(4): 531-6.

8. Polson J, Lee WM. AASLD position paper: The management of acute liver failure. Hepatology 2005; 41:1179-1197.

9. Arif S, T.A. Mufti, B. Iftikhar, S. Alam, A.S. Khan, J. Rehman and A.J. Safia. A study of prothrombin time and activated partial thromboplastin time in liver cirrhosis. JPMI 2005, 19(4): 495-498.

10. Mahmoud Mohamed Elgari. Evaluation of Hemostatic Parameters of Sudanese patients with liver Disease attending Khartoum Teaching Hospital. Egypt. Acad J Biolog Sci. Vol.5 (1) 1-5 (2013)

11. Hameed A, S. Naeem, A. Shaikh, A.S. Khursheed, A. Hamid and I.A. Naveed. An Assessment of coagulation parameters in liver cirrhosis. Biomedica 2006, 22: 74-77.

12. Bernardl M, G. Palareti, P. Pint, G.C. Caletti, E. Brocchi and G. Gasbarrini. Study on coagulation profile of patients with cirrhosis of the liver undergoing elective fiberoptic injection sclerotherapy oesophageal varices. Hepatogasteroenterol 1984.31: 125- 28.

13. Chisholm M. Haematological disorder in liver disease: In Sadler G.H.M. Wright R. Arthur MJP eds. Wrights' Liver and biliary disease. 3rd eds 1992. London. W. B. Saunders Company, 217: 22-24.

14. Tomiya T. Fujiwara K. Plasma thrombin Antithrombin III complexes in the diagnosis of primary hepatocellular carcinoma complicating liver cirrhosis Cancer 1991; 67:481-5.

15. Lee S. Yip M. Sachs H.J. Factor IX deficiency in liver disease. Journal of American Medical Association.1972: 221: 1410-12. 


\section{ORIGINAL ARTICLE}

\section{AUTHORS:}

1. Chikkalingaiah

2. Keshava H. K.

3. Prakruthi J.

\section{PARTICULARS OF CONTRIBUTORS:}

1. Professor, Department of Medicine, Kempegowda Institute of Medical Sciences and Research Institute.

2. Assistant Professor, Department of Medicine, Kempegowda Institute of Medical Sciences and Research Institute.

3. Post Graduate, Department of Medicine, Kempegowda Institute of Medical Sciences and Research Institute.

\section{NAME ADDRESS EMAIL ID OF THE} CORRESPONDING AUTHOR:

Dr. Chikkalingaiah,

No. 13, $4^{\text {th }}$ Main,

Hebbal, Bangalore - 560024 .

E-mail: prakruthi30@gmail.com

dr.chikkalingaiah@gmail.com

Date of Submission: 13/02/2014.

Date of Peer Review: 14/02/2014.

Date of Acceptance: 22/02/2014.

Date of Publishing: 06/03/2014. 